\section{Studien über Fleckfieber.}

\section{Von Dr. L. Finkelstein in Kowno.}

Die nachstehende Arbeit ist eine Zusammenfassung von Beobachtungen an etwa 900-1000 Fleckfieberkranken, die von mir, als äiterem Assistenzarzte des ukranischen Nr. 10/469 Reservespitals zu Proskurow (Podolie11) und später stellvertretendem Hauptarzte des Proskurowschen Semstwo-Infektionsspitals, in den Jahren 1919 bis 1920 behandelt wurden.

Die unerhörte Fleckfieberpandemie, die in der Ukraine und Rußland in den Jahren 1919 und 1920 wütete und auch jetzt noch nicht gallz erloschen ist, wurde zuerst all den Grellzstationen längs der galizischen Grenze, durch die die Massenrïckwanderung der Miiilärgefangenen aus Oesterreich-Ungarn nach Ausbruch der Revolution stattgefunden hat, bemerkt. Die ersten Fälle wurden nicht diagnostiziert, weil man in der schrecklichen - Grippenepidemie stand und die Spitäler dermaßen überfüllt waren, daß von einer individuellen Behandlung der Kranken keine Rede sein konnte (so mußte z. B. ein Spital, das 120 Betten hatte, 550 Krauke aufuehmen, und 2 Aerzte solltell die 550 Schwerkranke11 behandeln). Der Krieg zwischen Petlura und den Bolschewiki, wobei besollders die Ukrainer kein Versländnis für epidemiologische Prophylaxe hatteı, gab den passendell Nährboden für die Verbreitung der Epidemie, und dies, in Verbindung mit dem niedrigen Kulturniveau der Bevölkerung, führte z11 einer solchen Pandenlie, daß fast kein Haus von ihr verschont blieb und etwa 60-70\% der gesamten Bevölkerung, die Krankheit durchmachten.

Ehe wir zum Kranklıeitsbild übergehen, möchtelı wir über einige Fälle berichten, die ihrer Seltenlıeit wegen der Erwälınung wert sind. So saliell wir einen Säugling, 5 Monate alt, der ein regelrechtes Fleckfieber durchgemaclit hat, mit großem Exanthem, das den ganzen Körper bedeckte, und liohem Fieber. Auch einige Fälle von kombinierter Erkrankung, gleichzeitig Fleckfieber und Febris recurrens, kamen uns zu Gesicht. Nachdem die Spirochete Obermeyeri im Blut gefundell worden, machten wir in jedem Falle eine intravenöse Neosalvarsaninjektion; wir sahen gewöhnlich nach $20-24$ Stunden einen Abfall der Temperatur, aber bald darauf stieg die Temperatur wieder in die Höhe, und die Patienten macliten einen typischen Exanthematikus durch. Diese Fälle scheinen uns ein theoretisches Interesse insofern zu haben, als sie der Annahme eiliiger Autoren, der Erreger des Fleckfiebers sei ein Protozoon, strickt widersprechen.

Das Krankheitsbild. a) Die Initialperiode.

Der Anfang kann zwar schleichend sein, jeder intelligente Patient aber kann den Augenblick bezeichnell, wenn er Kopf- oder stechendell Brustschmerz (das habe ich persöulich erlebt) oder Frösteln zuerst empfunden hat. Die Temperaturmessung am ersten Tage hat $\mathrm{nie}$ mehr'als $37,4-37,9$ ergeben. Am zweiten Tag steigt sie am Abend gewöhnlich schon auf 39,4-39,8. - An den Organen findet man nichts Abnormes, manchmal eine Angina catarrhalis, etwas belegte Zninge. Am dritten Tage, der uns der charakteristischste Tag der Initialperiode zu sein scheint, kann man oft zwei Tatsachen, die den Arzt an Fleckfieber zu denken veranlassen, feststellen: 1. Eine bedeutende Temperatursenkung am Morgen des dritten Tages, die nur 5-6 Stunden anhält; am Abend erreicht die Temperatur wieder die frühere Höhe; gewöhnlich mißt man am Morgen des dritten Krankheitstages eine Temperatur von $37,4,37,6,38^{\circ}$, in vier Fällien sahı icl s ooga rinormale Temperatur. Diese Senkungszacke". die in der literatır beschrieben ist und von uns schon im Anfang der Epidenie tür die Frühdiagnose des Fleckfiebers verwertet wurde, sahen wir immer am dritten $\mathrm{Tage}$; sie scheint uns für Fleckfieber patlognomonisch zu sein, den in keinemeinzigen Falle, wo die Tempuraturmessungen vom ersten Moment der Erkrankungen sorgfältig registriert wurden (was sehr selten geschieht, denn die ersten Tage werden meistens übersehen), ve rmiBten wir s.i e. Natürlich kann diese Temperatursenkung nur dann von Bedeu. tung sein, welln keine Antipyretika gegeben wurden, was infolge des AspirinmiBbrauches recht selten der Fall ist.

2. Eine merkliche Verdumpfung der Herztöıle. besonders an der Herzspitze, schon am Abend des dritten Tages. Diese Verdumpfung vermift mant nur in leichten Fällent. - Manchmal (in etwa 15\% der Fälle) findet man am dritten bis vierten Tag eine vergrößerte, harte, nicht druckempfindliche Milz.

b) Exanthem. Am vierten, öfter am fünften Tage findet man die ersten Roseolen; seltener sieht man den Anfang des Exanthems am sechsten bis siebenten Tag; in zwei Fällen sahen wir das Exanthem erst am neunten Tage erscheinen. Meistens benutzt man die ersten Roseolen an dell Vorderarmell, nahe den Handgelenken, an den Handrücken und Handteliern, manchmal zwischen den Schulterb!ättern, am Kreuz. In 2-3 Tagen hat das Exanthem seinen Höhepunkt erreicht, und schoii bleichen die ersten Roseolen ab, währénd an anderen Stellen sich eine petechiale Umwandlung der einzelıen
Roseolen vollzieht. In schweren Fällen sieht man schon am siebenten bis achtell Tag zahlreiche Petechien, die dell ganzen Körper bedeckell und zum neunten bis zehnten Tag eine zyanotische Verfärbung aunehmen (was prognostisch voll sehr übler Bedeutung ist). In mittelschwerelı Fällen sitıd schon an zelınten Tage alle Roseolen verscliwundein, und nur eine mäßige Anzahl dunkelroter Petechien ist am Körper zu sehen. In leichten Fällen ist das Exanthem so flüchtig, daß es der Arzt leicht übersehen kanıl, und das hat wahrscheinlicl zu der Bezeichnung „Febris exanthematica sine exanthemate“ gefiihrt. Sehr scliwer ist der Ausschlag bei malträtierter Haut zu sehen: bei postskabiösen Ekzemen, zwischen reichlichen Pigmentierungell, und auch bei Leuten mit dunklem Teint der Haut, z. B. be Zigeunern, Beßarabienı usw. - Was das Exanthem in seinem Verliältuis zur Sclıwere des Krankheitsverlaufs anbetrifft, so hat sich uns der folgende Satz bewährt: je umfangreicher das Exanthem ist, je schueller und in größerer Zalı1 die Petechialisierung der Roseolen vor sich geht, um so schwerer verläuft der Fall, am so schwerer ist die allgemeine Intoxikation, um so schlechter ist die Prognose.

c) Temperatur. Abgesehell von der Senkungszacke am dritten Tag, ist die Temperatur fast immer eine Kontinua. Werte über $40^{\circ}$ sind recht : selten, gewöhnlich pendelt die Temperatur zwischen $39^{\circ}$ und $399^{\circ}$; nur in der vorkritischen Periode hat sie eine Tendenz, sporadisch am Abend auf $40,2-40,4$ zu steigen; dabei steigt gewöhnlich die Pulszahl nicht mit in die $\mathrm{Höhe}$, und dieses benigne Mißverhältnis von Pulszahl und Temperatur hat, wie wir weiter sehell werden, eine gute prognostische Bedeutung. Abweichungen von diesem Temperaturtypus kommen vor. Typhusäln11liche Kurven sieht man recht selten; Fleckfieber mit Temperatur, die kein einziges Mal $38^{\circ}$ erreichte, sah ich eilumal bei einem älteren Menschen; dieser Fall verlief selır schwer und endete tödlich. Anderseits gibt es Fälle, wo der Temperaturanstieg sehr langsam erfolgt, die sehr leicht verlaufen, aber sich bis zum 21 bis 22. Tage hilziehefi, onne irgendeine Komplikation. Der Temperaturabfall geschieht lytisch, im Laufe von zwei bis drei Tagen, unter schwacher Schweißabsonderung (manchmal aucli ohne SchweiB). Die abgeschlossene Temperaturkurve ist so charakteristisch, daß man mit ihrer Hilfe eine retrospektive Diagnose allf Febris exanthemica auch dann stellen darf, wenn kein Exanthem zu beobachten war.

d) Herz und Blutgefä Be. Wir haben es von den ersten Tagen der Krankheit ab mit einer akuten toxischen Myokarditis zu tun. Die Verdumpfung der Herztöne, besonders an der Herspitze, die scholl vom dritten bis vierten Tage an bemerkbar ist, steigert sich mit jeden Tage, um am achten bis zehnten $\mathrm{Tag}$ ihr Maximum zu erreichen. Der Puls, manchmal im Anfang relativ verlangsamt, wird mit jedem Tage schlechter: die Pulskurve steigt unaufhaltsam in die Höhe; obwohl die Temperatur eine Kontinua ist; der Puls wird immer weicher. schlechter fühlbar, ist leicht zu unterdrücken, irregulär in der Füllung (eine Art Pulsus alternans), aber behält gewöhnlich den richtigen Rlıythmus. Die Pulszahl steigt bis $130-140$ Schläge in der Minute (bei Frauen allch über 150). Im Laufe des zehnten bis zwölften Tages erfolgt die EntscheidungderFrage ob das gescliilderte Anwachsen der Herzschwäche durch Einsetzen der Reservekräfte des H'erzmuskels halt macht oder aber es fortschreitet, um am 15. bis 16. Tage in Erlahmung überzugehen. Während dieser Tage sehell wir in benign verlaufenden Fällen einen richtigen Umschwung in der Herztätigkeit: zuerst eine kleine Aufhellung der Herztöne (auf der Pulskurve gleichzeitig ein Stillstand in der Aufwärts- resp. eine kleine Abwärtsbewegung), dann werden mit jeder Stunde die Herztöne reiner, die Pulsqualität resp. Zalıl verbessern sich (auf der Pulskurve sclion eine ansehnliche Abwärtsbewegung - um 4-8 Schläge in der Minute). Das alles läßt sich $11 / 2-2$ Tage vor dem Anfang des Temperaturabfalls, ja gewöhnlich gleichzeitig mit einer Temperatırerlıöhung feststellen. Und wenı man von einer Temperaturkrisis bei Fleckfieber nicht reden kann, so kann man mit vollen Recht von e iner Herz-bzw. Pul'skris is sprechen die uns einc feste prognostische Stiitze zu einem Zeitpunkt gibt; wo die Temperatur noch sehr hoch, das Bewußtsein schwer getrübt ist und das allgemeine Bild geradezu hoffnungslos aussieht. Sahen wir bis zum Ende des zwölften Tages diese Herz- bzw. Pulskrisis nich eintreten, so war es immer ein Zeiche11, daß der Herzmuskel über keine Reservekräfte verfügt, und diese Fälle verliefen alle tödlich

-Arhythmien sahen wir während der febrilen Periode seiten (das waren Patic:liten mit alten Myokarditiden); viel öftér sahen wir extrasystolische Arhythmien während oder unmittelbar nach der Entfieberung; manchmal war die Arhythmie sehr stark (Ausbleiben eines Pulsschlages nach jeden 2-3 Sch'ägen); dabei blieb aber die Pu'sfiillung gut und die Herztöne, abgesehen von der Rhythmusstörung, reili. Diese spãten Arhythmien haben eine gute Prognose.

4 Fälle von Fleckfieber bei Patienten mit kompensierten Herzklappenfehlern (alle Mitralisinsuffizienz), die wir beobachtet haben, verliefen nicht schwerer als andere aus derselben Reihe; in einem dieser Fälle wurden während der Rekonvaleszenz recht starke Herz schwächeanfälle beobachtet. Solche Anfälle von Herzschwäche sind anch bei Patienten mit früher gesundem Herzen während der Rekonvaleszeiiz gesehen worden. Manchmal sieht die Herzschwäche recht bös aus; man möchte fast von, einem Kollaps sprechen. Jüngere Patienten erholen sich aber im Laufe von ein bis zwei Tagen wieder, ältere 'können daran zugrundegehen.

e) A tmungsorgane. Eine gewöhnliche Bronchitis gehört zum Krankheitsbild. Je stärker die Herzschwäche ausgeprägt war, "um 
so öfter saleıl wir diese harmlose Bronchitis in eine Pneumonie übergehen; je frühzeitiger die Pneumonie entstand, um so trüber war die Prognose, denn der schwer intoxizierte Herzmuskel war innerhalb der erstell acht bis zwölf Krankheitstage dieser neuen Aufgabe nicht gewachsen. Hingegen verliefen die späten Pneumonien (vom 13.-15. Tage an) viel gutartiger. Es scheint uns, daß in der Aetiologie der Prieumonien bei Fleckfieberkranken eine sehr wichtige Rolle neben der Herzschwäche auch der Umstand spielt, daß die schwer benommeneı Patienten sehr oberflächlich atmen.

Nicht seltell sahen wir im Anfang der Rekonvaleszenz exsudative Pleuritiden; sie hatten keine Tendenz zur Vereiterung; die Temperatur stieg nicht höher als $37,5^{\circ}$; manchmal klagten die Patien. ten über Seitenstecheıl. Unter kleinen Salizyldosen trat gewöhnlich in acht bis zehn Tagen eine vollkommene Resorption der Ergüsse ein. abwenden. Gleichmäßige Temperatur des Krankenzimımers, ofter Lagewechsel, gute Mundpflege spielen die wichtigste Rolle. Eine Verminderung der Immunität des Organismus gegen Eiterungen liegt dell häufigen Eiterungen zugrunde - A bs zessen, Phleg mone n: und den gefürchteten Par otitiden. Hierher gehört auch die Phle bitis. Gangrän, von Zehen- bis Extremitätengangrän;" war nicht selten in Spitälern, besonders im Winter 1919, zu sehen, als infolge chaotischer politischer Zustände die Holzzufuhr sehr dürftig war. Einmal erlebten wir eine Apoplexie mit. tödlichem Ausgang, und einmal eine einseitige Rekurre 1 slähmung.

k) The ra pi e. Ebenso wie man bei kruppöser Pneumonie frühzeitig mit der Digitalistherapie eilusetzt, ist es auch ein Gebot beim Fleckfieber, s of ort nacli Klarwerden der Diagnose zur Digitalistherapie $z u$ schreitell. Am besten hat sich uns

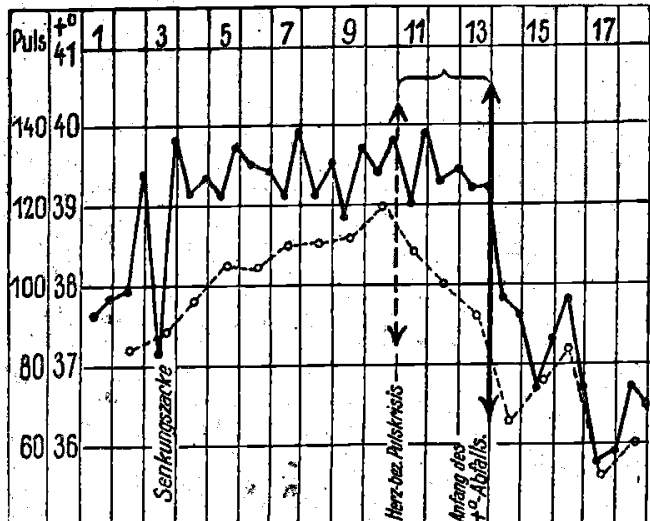

Dr. K. A., 29 Jahre
Lelchter Fall.

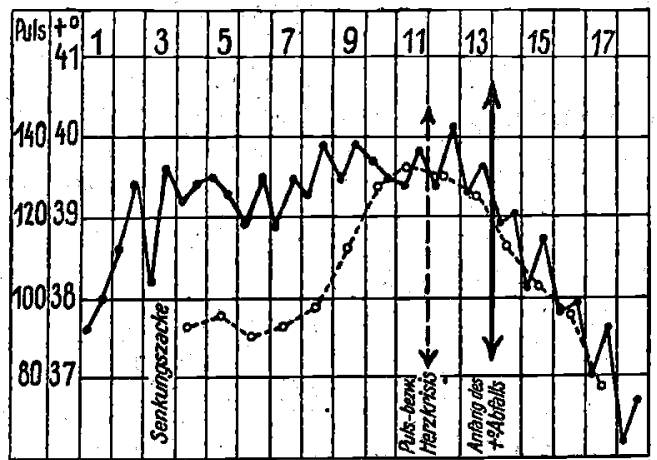

L. G., 41 Jahre.

Mitteis chwerer Fall.

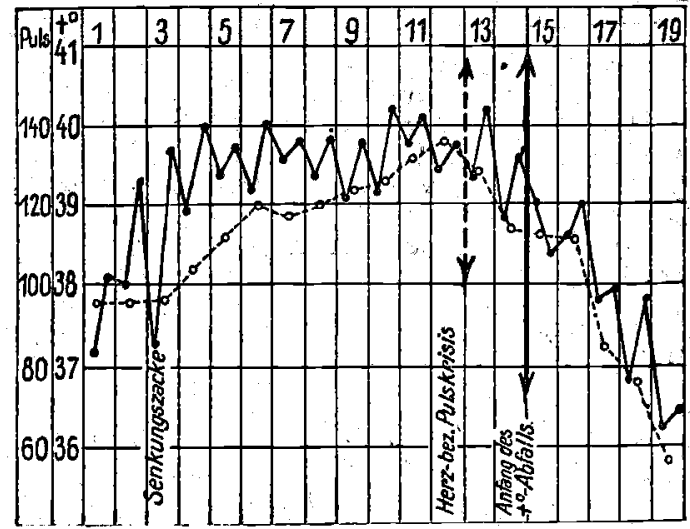

Maria P., 23 Jahre.

Schwerer Fall.

f) Nervensystem. Im allgemeinen geht die Beeinträchtigung des Nervensystems parallel der gesamten Intoxikation; aber einerseits sahın wir schwerste tödliche Fleckfieberfälle fast ohne. Delirieı und anderseits klinisch leichte Fälle mit äußerst heftigen Tobsuchtsanfällen, mit nachfolgenden Psychosen. Diese Psychosen dauerten drei bis vier Wochen an und verliefen gut, ohne die allgemeine Restitutio zu benachteiligen. Es scheint uns eher die allgemeine nervöse Konstitution des Patienten für die Stärke der nervösen Reaktion verantwortlich zu sein als die Stärke des Virus: bei leicht erregbaren Leuten mit beweglichem Temperament sahen wir ofter Delirien und Erregungszustände als. bei apathischen, zu Depression neigenden, bei denen viel stärker die Schlaflosigkeit iı den Vordergrund trat.

Eine recht schlechte Prognose geben Sprachstörungen, besonders weni sie frühzeitig auftreten: erschwerte, skandierende, schlecht artikulierte Sprache. Singultus ist ein quailendes und ein recht ernst zu nehmendes Symptoin, aber nicht inımer von infauster Prognose: in eiinem Falle, wo der Singultus den Patienten sechs Tage quälte, trat doch schließlich Besserung und Genesung ein.

Störungen des Geliörs, Olırensausen und zeitweilige Taubheit kommen so oft vor, daß man sie fast zuin Krankheitsbild hinzurechnen möchte. Die Taubheit lıält eine bis zwei Wochen an und schwindet spurlos; gewölnnlich wird sie in der zweiten Krankheitswoche bemerkt und geht in die Rekonvaleszenzperiode über.

g) Magendarmtraktus. Recht oft ist in der ersten Krankheitswoche bei. Frauell Uebelkeit zu beobacliten, die zu Erbrechell fülıreı kanı. Eine starke Salivation, wobei die Mundhöhle immer voll schleimigen Speichels ist und die den Patienten furchtbar quälen kaun, ist nicht selteil zu sehen. Der Stuhl ist in den meisten Fällen augehalten in etwa 10-15\% der Fälle sind hingegen Durchfälle $z u$ verzeichnen; stärkere Grade des Durchfalls, die zu großen Wasserverlusteı führen, verschlechtern die Prognose.

h) Nieren. Fast immer (mit Ausnahme der leichtesten Fälle, Fanden wir im Urin recht viel Eiweiß (bis $9 \%$ gewöhnlich aber $1-2 \%$ ) und im Sediment hyaline und granulierte $Z$ ylinder in großer Zahl, manchmal auch Erythrozyten. Auch hier war der Paralle'ismus zwischeıl Schwere der allgemeineıı Intoxikation und Nierenschädigung unverkennbar: je schwerer der Fall, um so pathologischer die Harnzusammensetzung. In schweren Fällen haben wir eine akute Nephritis vor uns und keineswegs nur eine Albuminurie, wie es so häufig gesagt wird. Sofort nach Verbesserung der Herztätigkeit kann man schon ein Besserwerden der Diurese und der Harnbeschaffenheit feststellen, und acht bis zehn Tage nach dem Temperaturabfall ist schoil von der Nephritis nichts zu sehen.

Nicht selten sahell wir eine Erschwerung der Harnentleerung. Gewöhullich genügte die Anwendung einer Wärmflasche (auf die Gegend der Harnblase), um dem abzuhelfen, und nur selten mußte man katheterisieren.

i) Komplikatione n. Im allgemeinen sind die Komplikationen das Resultat einer nichtindividuellen Krankenpflege, und darum sind sie so häufig. in schlecht organisierten Krankenhäusern und recht selten in der Privatpraxis. Obwohl als Ursache der verschiedenen Komplikationen die von den Pathologen festgestellte Arteriitis (mit Lokalisation in den präkapillären Gefäßen und Neigung zu Wandnekrose und Thrombose) anzusprechen ist, kann doch eine aufmerksame, individuell angepaßte Pflege des Patienten manches drohende Uebel die folgende Vorschrift bewährt: Inf. f. Digitalis e 0,6:180,0; Natr bromati 4,0 (5,0); Codeini ph. 0,2 (0,1). Dreistündlich einen Eßlöffel. Der Schlaf wird ruhiger, Kopfschmerzen etwas geringer. Drei Flaschen dieser Mixtur reiclien gewöhulich für die Zeit vom sechsten bis zwölftell Tag aus. Wird die Herztätigkeit bis zum zwölften Tage merklich besser, so gibt man von diesem Tage all statt dieser Mixtur Tinct. Strophanthi, Tinct. nuc. vomic. āã $8,0-$ dreimal täglich 15 Tropfen bis zum Ende des Temperaturabfalls. Wenn aber keine Besserung eiılgetreten ist, so gibt man jetzt. Digalen intravenös 2-3mal täglich manclımal noch init Erfolg. In schwereı Fällen kann man auch Kampferöl- resp. Koffeininjektionen hinzufügen.

Eille eilımalige Gabe von Pyramidon 0,3 kann bei heftigen Kopfschinerzell von Nutzell sein; eille Pyramidontherapie haltell wir für koutraindiziert da sie nur die Herztätigkeit schwächen kann. Bäder von $28^{\circ} \mathrm{R}$. können bei Erregungszuständen gut wirken, aber auch Bäderthe rapie ist $z u$ verwerfell, da es schwer ist, . Bäder $z u$ machell, olı1e den Patienten durch Bewegungell zu strapazieren. Wir legen größten Wert auf absolute körperliche Rulıe des Patienten, da wir wiederholt Kollapse nach unvorsichtigem Transport des Kranken ins Krankenhaus (man ließ ihn z. B. eille Treppe steigen) oder nach einem Bade gesehen haben. Bei kühl sich anfülılenden Füßen soll man sofort Wärmeflaschen anweliden.

Subkutaile Infusionen von physiologischer Kochsalzlösung mit und ohne Adrenalinzusatz haben wir viel verwendet. Als Indikation dazu möchten wir größere Wasserverluste des Organismus ansehen starke Durchfälle, Neigung zum Schwitzen u. a.m.

Zusammmenfassung. 1 . Als eine Gefäßerkrankung schädigt das Fleckfieber durch die Blutgefäße alle Organsysteme des Organismus, in erster Linie das Herz, das Nervellsystem und die Nieren.

2. Die frühe Herztöneveränderung und die Temperätursenkungszacke amı dritten Kranklıeitstage scheinen für die Frühdiagnose des Fleckfiebers von Wert zu sein.

3. Die ersten Zeichen der eingetretenen Intoxikationsverminderung m Laufe der Krankheit dokumentieren sich in Laufe des zehnten bis zwölften Krankheitstages: a) in einer Herztöneaufhellung, b) in einer Verminderung der Pulszahl und c) in einer Verbesserung de Pulsqualität (Herz- bzw. Pulskrisis des Fleckfiebers) darauf folgt Verbesserung der Diurese und der Harnbeschaffenheit und als letztes Glied der Kette die langsame Entfieberung.

4. In der Therapie des Fleckfiebers nimmt die frühzeitige Digitalisverwendung den wichtigsten Platz ein. 